\title{
Biomedical and Biochemical Tools of Förster Resonance Energy Transfer Enabled by Colloidal Quantum Dot Nanocrystals for Life Sciences
}

\author{
Urartu Özgür Şafak Şeker and Hilmi Volkan Demir
}

\section{Contents}

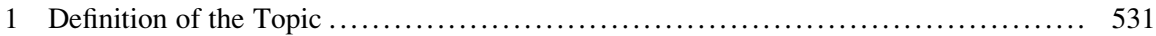

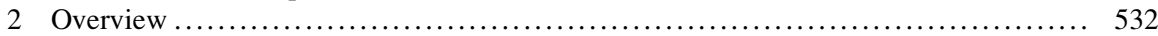

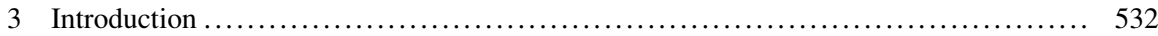

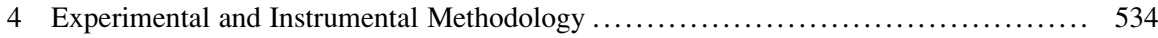

$4.1 \quad$ FRET Using Quantum Dot Nanocrystals ............................... 534

4.2 Characterization Methods of the FRET Process ........................... 538

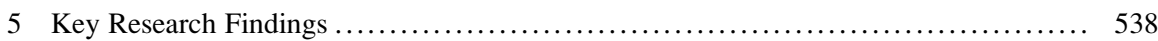

5.1 Quantum Dot Nanocrystals Enabled Biosensors .......................... 538

5.2 Quantum Dot Nanocrystals Enhanced Bioimaging ......................... 551

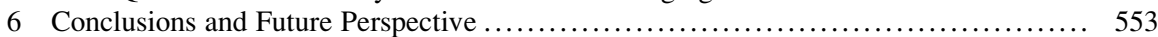

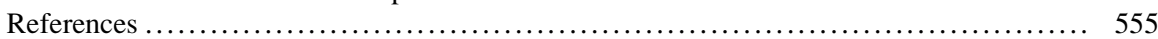

\section{Definition of the Topic}

Semiconductor quantum nanocrystals (NCs) provide the ability to control and fine-tune peak emission wavelength using the size effect, with a broad optical absorption band (excitation window) increasing toward UV wavelength range. Quantum dots with different peak emission wavelengths can be excited at the same wavelength and offer longer fluorescence lifetimes, which make them desirable donor molecules for Förster resonance energy transfer (FRET)-based applications. In this chapter, the tools of FRET using these quantum dot nanocrystals in life science applications are addressed.

\footnotetext{
U.Ö.Ş. Şeker $(\bowtie) \bullet$ H.V. Demir

Department of Electrical and Electronics Engineering, Department of Physics and UNAMInstitute of Materials Science and Nanotechnology, Bilkent University, Ankara, Turkey

Luminous! Centre of Excellence for Semiconductor Lighting and Displays, School of Electrical and Electronic Engineering, School of Physical and Mathematical Sciences, Nanyang Technological University, Singapore, Singapore
} 


\section{Overview}

Förster resonance energy transfer arises from the dipole-dipole interaction of donors and acceptors in their close proximity (typically less than $10 \mathrm{~nm}$ ). FRETbased applications have been widely used in life sciences for a long time, especially utilizing fluorescent proteins and organic dyes as the donors and/or acceptors. FRET-based biological applications range from biosensing of a target molecule to the imaging of the transportation of a molecule within a cellular compartment. With the recent developments and improvements in nanotechnology, quantum dot nanocrystals have become the next generation fluorophores with their size-tunable emission properties and broadband absorption properties.

Quantum dot nanocrystals can be synthesized using both solvent and aqueous synthesis approaches. To conjugate the quantum dot nanocrystals with biomolecules and dye acceptors, surface functionalization is crucial. The surface functionalization may be carried out using a number of different approaches. The most popular chemical approach is the formation of carboxyl groups on the quantum dot surface by adding ligands during synthesis and activation of the carboxyl groups using carbodiimide chemistry based on NHS ( $N$-hydroxysulfosuccinimide)/EDC (1-ethyl-3-(3-demethylaminopropyl)carbodiimide) activation. Another approach for the biomolecule conjugation to quantum dots relies on using conventional streptavidin-biotin interaction. Also, metal-affinity-based conjugation of biomolecules, especially proteins, is of great interest because of the simple application of this method. Thus far the protein-, peptide-, and DNA-conjugated quantum dots have been utilized in FRET applications for biosensing and imaging techniques.

In this chapter, we present how to utilize biomolecule-quantum dot conjugates in FRET applications for life sciences, which promise a number of new possibilities for biosensing and imaging.

\section{Introduction}

To track intracellular and extracellular events, many different labeling techniques have been employed. These include labeling the target biomolecule using a fluorescent reporter. This fluorescent molecule can be either another biological molecule such as a fluorescent protein and peptide, or more commonly a chemical dye molecule [1, 2]. Many dye molecules have been successfully designed, synthesized, and attached to certain biomolecules to track changes and processes in intracellular and extracellular environment [3, 4]. Many of these dye molecules have also been successfully utilized to make biochemical assays to determine the amount of a given molecule quantitatively and qualitatively [4, 5]. Although these approaches worked well in most cases, there have been certain limitations, which in turn make their potential limited for understanding the biological complexity and underpinning mechanisms. Most of the dye-protein labeling approaches need to be normalized with respect to the number of molecules used in the extracellular assays. This makes it hard to follow the changes upon interacting a labeled molecule, 
as there is only one signal but many possible interactions. For biosensing applications, using only one labeled species can be problematic to track changes upon molecular interactions. Following up a single signal can cause nonspecific interaction errors. Considering all these factors, a more powerful technique, Förster resonance energy transfer - FRET, has found a large area of usability in biolabeling applications [6]. Förster resonance energy transfer, also sometimes referred to as the fluorescence resonance energy transfer in the community, is a phenomenon that takes place through the nonradiative transfer of the electronic excitation energy from an electronically excited donor to a fluorescence acceptor, which can only occur if the distance between the donor and acceptor species is smaller than twice Förster radii (typically corresponding to a distance of less than $10 \mathrm{~nm}$ ) and if the emission of the donor spectrally matches the absorption of the acceptor. This phenomenon was first explained by Theodor Förster in 1948. Since then, FRET has been widely used in biological and biochemical applications, among which are protein-protein interactions, conformational change of proteins, DNA hybridization, and receptor-ligand binding [7].

For the protein-protein interaction studies, if the interaction is being carried out in an intracellular environment, generally both of the molecules are fused with a fluorescent protein such as green fluorescent protein (GFP) and red fluorescent protein (RFP), and through the change in FRET signal, the strength of the interaction can be quantified. Mostly, the FRET signal in intracellular protein-protein interaction type of these studies is monitored under fluorescence microscopy. Similarly, another approach relies on labeling both of the interacting molecules using conventional dye molecules that possess a good spectral overlap for FRET application. These approaches can be useful especially for extracellular FRET-based bioassays.

One of the popular utilization of FRET signal is for monitoring changes in the structure of a protein. In this approach, a protein molecule must be labeled in a sitespecific manner with suitable fluorescence dyes, because any structural change that results in the folding of the protein will lead to a distance change between the dye molecules, and as long as the separation between the dye molecules satisfies the FRET conditions, one can easily correlate the structural rearrangement of the protein with the FRET signal. This event can be calibrated with reference molecules in such a way that FRET signal can be used as a molecularly sensitive nanoruler [8]. Similarly, upon the denaturation-renaturation of the proteins, the distance between the fluorescent labels can be changed, which can be followed through the change in the FRET signal [9]. Ligand-receptor binding was also characterized using FRET processes, where both ligands-receptors were labeled using fluorescent dyes [10]. This approach is now also widely utilized to analyze the molecular interaction in biological systems.

FRET applications have been carried out using a range of different materials dominantly including organic dyes, natural fluorophores (tryptophan, tyrosine, and phenylalanine), and fluorescent proteins, and enzyme catalyzed bio- and chemoluminescence reactions were also used [11-13]. However, one of the problems in most of these applications was the short fluorescent lifetime of the given molecules and materials, for which some other alternative dyes and materials were utilized [6]. 
The number of recent studies conducted to use nanoparticles in FRET studies is also increasing. Mostly metal- (e.g., gold) and semiconductor- (e.g., silicon) based nanoparticles as well as luminescent quantum dot nanoparticles have been used for their exceptional size-tunable properties [14]. With these, innovative improvements in nanotechnology, especially quantum dot nanocrystals, have been investigated to replace the currently available organic dye molecules. Compared to the organic dye molecules, these quantum dots have superior characteristics such as the sizetunable photoluminescence, relatively sharp emission spectrum, broad adsorption spectrum, and large Stokes shift, which make their emission band far from their absorption edge [15-17]. Using these unique properties of the quantum dots, they can be tuned to provide stronger spectral overlaps with a certain type of organic, natural, or inorganic acceptor. Today, many quantum dot nanocrystals, which can be utilized as FRET donors, are available in the market with a wide range of peak emission wavelength options. Also, the synthesis of these quantum dots can be easily achieved using the simple well-established recipes [18].

The applications of the quantum dot nanocrystals in biological and biomedical research have been covered in some other previous review papers and book chapters. However, in this chapter, rather than a general overview of the applications of the quantum dot nanocrystals, a more focused route into the FRET-based applications of the quantum dot nanocrystals is considered. This focused coverage aims at providing a rich source on the quantum dot-enabled FRET-based applications carried out for life sciences in the literature. This is especially important for a researcher in the biomedical and biotechnological research areas to envision novel applications exploiting quantum dot-based FRET as a powerful tool.

This chapter aims to cover the utilization of quantum dot-enabled FRET in biomedical and biochemical applications. In this sense, we are giving an overview starting from the basics of the quantum dots to the advanced applications of quantum dot-enabled FRET in life sciences by covering latest literature. This chapter starts with the synthesis and surface functionalization of the quantum dot nanocrystals, which is the key concept for their utilization in FRET-based processes. Subsequently, the use of these quantum dots is presented for FRET applications under two main titles, which are the most common applications of FRET processes: the biosensing and the bioimaging. Having different target molecules and utilizing different types of molecular recognition elements, biosensing applications are grouped as the antibody, protein, and nucleic acid-based applications.

\section{$4 \quad$ Experimental and Instrumental Methodology}

\subsection{FRET Using Quantum Dot Nanocrystals}

\subsubsection{Semiconductor Quantum Dot Nanocrystals Synthesis}

The first step toward the discovery and realization of the solution-phase formation of the colloidal quantum dots was taken just after the demonstration of the quantum confinement effect in semiconductor crystals. It was found that semiconductor 
nanocrystals can be formed within the micellar structures. This first approach included the synthesis of the semiconductor nanocrystals at the water-oil interface using a surfactant. The researchers showed that by addition of the metal salts, one can easily synthesize the quantum dots within the room temperature limits; however, these initial attempts led to the formation of nanocrystals with poor crystalline structures in the early times [19].

Quantum dot nanocrystals are made of many different semiconductor materials, e.g., CdSe, $\mathrm{ZnSe}, \mathrm{CdZnSe}, \mathrm{PbS}$, and InP, just to name a few. Later after the initial attempts for the synthesis of the quantum dot nanocrystals, Bawendi et al. demonstrated that high-quality quantum dot nanocrystals with high quantum yield and narrow size distribution can be obtained through a hot injection synthesis at high temperatures, which is considered as a revolutionary milestone in the synthesis of quantum dot nanoparticles [20, 21]. In this reaction scheme, the precursor materials which are organometallic precursors were injected into a boiling coordinating solvent, e.g., trioctylphosphine (TOP) and trioctylphosphine oxide (TOPO). This process results in the thermal decomposition of the precursor materials and the monomers form the first nucleus of the quantum dot nanocrystals. Following the nucleation by further addition of the precursor monomers from the solution, the crystal growth is achieved. In the mean time, further newly added monomers are incorporated onto the existing nuclei instead of forming new nuclei because of their lower unsaturated concentrations. As soon as the monomer concentration is decreased to a certain level, the growth of nanoparticles continues the Ostwald ripening process. In this process, the smaller particles with higher surface tension may dissolve and contribute to the growth of larger particles. This continues until the system is saturated by the formed quantum dot nanocrystals.

The formation of quantum dot nanocrystals can be controlled by the addition of certain surfactants and ligand molecules. The longer the reaction continues, the larger particles are formed. Because the size controls the peak emission wavelength of the quantum dot nanoparticles, one can optimize the reaction time to obtain quantum dots emitting at a desired wavelength. Cleaning of the synthesized nanocrystals is also important to remove the unreacted reagents, as there might be a high amount of acids, metals, and amines left unreacted in the solution. The synthesized nanocrystals are typically characterized using transmission electron microscopy, x-ray photoelectron spectroscopy, and wide-angle x-ray diffraction spectroscopy. Besides these, optical characterization of the particles must be carried out using absorption and fluorescence spectroscopy [22, 23].

Water is the crucial medium for all life forms and biological materials. To use synthesized nanocrystals in aqueous environments, there are two methods: one is to synthesize the quantum dot nanocrystals directly in an aqueous environment and the other is through the ligand exchange of solvent-based quantum dots. The successful synthesis of the aqueous quantum dot nanocrystals made of CdTe was first achieved by Nozik and Welles. During their synthesis of CdTe nanocrystals, Nozik et al. used 3-mercapto-1,2-propanediol and hexametaphosphate as the stabilizing agents. Alternatively, Welles et al. introduced different thiols such as mercaptoethanol and 1-thioglycerol in their aqueous synthesis [24]. 
Capping CdTe nanocrystals with TGA also makes it easy for their surface modification. Later, using aqueous routes of nanocrystals, various materials were synthesized; CdSe, CdS, CdTe, and HgTe are some of the aqueous NCs. Also, by applying different capping agents, especially thiols, the quantum efficiency of aqueous nanocrystals was increased up to $40-60 \%$ range. CdTe is now the most typically used material to make aqueous quantum dot nanocrystals, which has found many applications because of its simple synthesis while avoiding the need for any further modification toward water solubility. CdTe nanocrystals are synthesized from a $\mathrm{Cd}$ salt, most popularly from $\mathrm{Cd}\left(\mathrm{ClO}_{4}\right) \cdot 6 \mathrm{H}_{2} \mathrm{O}$. In the aqueous synthesis of CdTe, the salt is dissolved in water and a proper amount of thiol is added for stabilization and the flask is continuously stirred to sustain homogeneity. The control of the $\mathrm{pH}$ of the solution is achieved through dropwise addition of $\mathrm{NaOH}$. In the mean time, the flask is heated for 30 min aerating with $\mathrm{N}_{2} \cdot \mathrm{H}_{2}$ Te gas is flown through the flask with the aid of $\mathrm{N}_{2}$ flow. At the first stage, the precursor of $\mathrm{CdTe}$ is formed, and at the second stage, the precursors are refluxed at $100{ }^{\circ} \mathrm{C}$ under open air conditions by the aid of a condenser. During the reflux, CdTe NCs start to grow. Utilization of $\mathrm{Al}_{2} \mathrm{Te}_{3}$ as the Te source can be an alternative way for the CdTe nanocrystal synthesis, which can be more convenient but more expensive. During the synthesis of the CdTe nanocrystals, short chain thiols (namely thioglycolic acid, mercaptoacetic acid, 2-mercaptoethylamine, and cysteamine) with functional groups (e.g., amine, carboxyl, and hydroxyl) have been utilized [19, 25, 26].

\subsubsection{Surface Modification of Quantum Dot Nanocrystals}

In order to utilize the quantum dot nanocrystals in FRET applications, strategies for the conjugation with biomolecules and with certain dye molecules are needed. The conjugation of the quantum dot nanocrystals allows the dye and quantum molecule to be kept in the close proximity, which satisfies the nonradiative energy transfer. Commonly used approaches for the attachment of the fluorophores to a quantum dot for FRET applications can be grouped as follows.

\subsubsection{Electrostatic Interaction of the Quantum Dot Nanocrystals with Fluorophores}

In this approach, the quantum dot nanocrystals interact with the fluorophores through positively charged-negatively charged group interaction. In their study, Sun et al. found that aqueous CdTe solutions to yield high FRET efficiency while interacting with a polymer named poly (vinylcarbazole) in a blend. The interaction between these species was achieved through electrostatic interaction of the negatively charged CdTe and the positively charged polymer [27] Similarly, Osovsky et al. investigated the FRET process between CdTe NCs which are modified with two distinct functional groups, namely, positively charged cysteamine and negatively charged thioglycolic acid [28]. Here, the biomolecules were coupled with the quantum dots through electrostatic interaction using the charged groups on the backbone of the biomolecules. Also, quantum dot nanocrystal sensor complexes built up using single-strand DNA and the attachment of proteins are among the examples of biomolecule-quantum dot nanocrystals hybrid systems formed through 
electrostatic interactions [29-33]. Although the electrostatic interaction is not very strong compared to metal affinity or chemical attachments, it is widely used for building FRET complexes using quantum dots because of the easiness of the method. However, if more stable structures are needed for FRET-operating systems, other approaches can be pursued.

\subsubsection{Chemical Attachment of FRET Partners to Quantum Dot Nanocrystals}

The outer shell surface of the quantum dots can be modified with amine or carboxylic acid groups, or during the synthesis of the quantum dot nanocrystals, these groups can be introduced. During the synthesis, thioglycolic acid can be used as the ligand at the outer shell and the quantum dots then become decorated with the carboxyl groups. The carboxylic acid group can be activated using the addition of EDC (1-ethyl-3-(3-demethylaminopropyl)carbodiimide). This reaction leads to the formation of $\mathrm{O}$-acylisourea intermediate, which can react easily with the amine groups on the fluorescent biomolecule or the dye and forms a stable conjugate. In this reaction to prevent the hydrolysis of another intermediate molecule, NHS ( $N$-hydroxysulfosuccinimide) can also be employed [34-36]. Amine-functionalized quantum dots can also be synthesized; for example, cysteamine can be employed as a capping agent, or via ligand exchange, the quantum dot nanocrystal surface can be decorated with cysteamine. In this case similar to the $-\mathrm{COOH}$ functionalization, the carboxyl-containing molecule can be activated, and quantum dot nanocrystal can be conjugated via amine coupling [37].

\subsubsection{Metal Affinity Tag Enabled Assembly}

Metal affinity tag is a commonly used peptide sequence for purification of the proteins using an affinity-based chromatography approach. The most common metal affinity tag is a hexahistidine molecule, which interacts strongly with the metal atoms. Nickel is the most widely used metal for its strong interaction with the imidazole groups on the histidine amino acids. However, it was also demonstrated that it can strongly interact with the semiconductor materials [38]. Especially fluorescence proteins and dye-labeled proteins tagged with 6xHis have been proven to form FRET couples with quantum dot nanocrystals. Park et al. investigated to synthesize thiolated nitrilotriacetate-capped $\mathrm{CdSe} / \mathrm{ZnS}$ quantum dot nanocrystals, which were then shown to successfully capture the hexahistidine peptide conjugated with TAMRA dye [39]. Boeneman et al. achieved the assembly of a fluorescent protein mCherry on the surface of CdSe/ZnS NCs using the metal affinity tag as the linker molecule, which was later utilized in a FRET process successfully [40].

Other specific interactions were also used to conjugate fluorescent biomolecules and dye-labeled biomolecules to the quantum dot nanocrystals; for example, biotin-streptavidin interaction was widely used [41-43]. Other protein purification tags can also be employed for coupling proteins to the quantum dot nanocrystals, glutathione S-transferase; FLAG and strep-tag are the most promising ones for creating a protein-NC FRET couple [44]. 


\subsection{Characterization Methods of the FRET Process}

FRET process can be easily monitored using available fluorescence spectroscopy and time-resolved spectroscopy instruments. In fluorescence spectroscopy, the optical intensities of the donor and acceptor were measured separately, and then the optical intensity level of the mixture facilitating FRET must be measured. However, because the optical intensity level heavily depends on the molar ratio of the donor and acceptor in the solution, the optical intensity of the fluorescence signal must be carefully normalized for the three cases mentioned above. In fluorescence spectroscopy, only the change in the optical intensity change of the donor and acceptor molecules can be tracked.

An additional approach is to measure the fluorescence lifetime change of the donor and acceptor molecules. In this approach using a time-resolved fluorescence spectroscopy (e.g., time-correlated single photon count systems), the emission kinetics of the donor and acceptor are monitored and the decay lifetimes are measured. This leads to a more robust measurement of the FRET process, because the measurement changes in the emission kinetics are not as sensitive to the amount of the material that exists in the medium as the steady state measurements [45].

A time-resolved fluorescence spectroscopy tool needs to be equipped with a gated or pulsed laser source emitting at a suitable excitation wavelength, which can be used to excite the fluorescent materials subject to FRET process in the substrate medium. In order to focus the laser beam, a lens system is used and the beam is passed through a polarizer. Subsequently, the light is passed through a monochromator with a suitable slit aperture, and the photons can be counted using a high-precision time-correlated single photon counting system in the case of time-correlated single photon count systems [26].

\section{$5 \quad$ Key Research Findings}

\subsection{Quantum Dot Nanocrystals Enabled Biosensors}

\subsubsection{Antibody-Antigen Interaction Using Quantum Dot Nanocrystal- Enabled FRET Processes}

Nanobiosensors have been developed and/or improved in the last decade with better sensing capabilities and lower limits of detections [46]. Quantum dot nanocrystals have been widely used as the signal-generating parts of these sensing systems. FRET process is the best way to harness the optical properties of QD photoluminescence (PL). Quantum dots are versatile donors for FRET applications, and they can generate very efficient FRET processes when they are coupled with a proper acceptor [7, 47, 48]. Today, many researchers are interested in using quantum dots as donors for FRET applications for their favorable optical properties, which are mentioned earlier. Especially high-quantum-yield (QY) ratios of these quantum dots make them popular for FRET applications because a high QY allows for detecting at longer FRET distances. Also, the sharp and size-tunable emission of 
the quantum dots allows minimizing the cross talk between the acceptor and donor emission wavelengths during measurements. Quantum dots are ideal for multiplexed FRET configurations as more than one quantum dot emitting at different wavelengths can be excited at the same wavelength without an overlap with their respective PLs [6]. One of the important requirements for the biosensors is specificity. In this respect, the quantum dots provide very large surface areas which enable them to be easily functionalized for recognition elements using a number of different conjugation methods [18].

Quantum dot-based FRET has been used in biosensing applications to monitor different types of specific biological and biochemical events. In biosensing studies, one of the most studied and well-established approaches is based on utilizing molecular recognition elements to detect specific molecules such as antibodyantigen systems. In an antibody-based sensing system, typically a sandwich type of assay is utilized to capture an antigen. Here, two antibodies with affinity to the same target can be used. In this case, the first antibody is labeled with a quantum dot, and the second antibody, reporter antibody, is labeled with a dye molecule [49]. If a target antigen is added into the solution, it binds both the antibodies, which finally creates a FRET-controlled quenching of the quantum dot with the dye molecule, and the loss in PL of the quantum dot depends on the concentration of the target antigen [50]. This assay configuration may, however, cause some problems including a possible heterogonous A-D separation due to the bigger size of the antibody, which can then result in mixed antibody avidity [51]. For example, Wei et al. tested this configuration by conjugating a monoclonal antibody specific for estrogen receptor $\beta$ (ER- $\beta$ ) to a quantum dot emitting at $565 \mathrm{~nm}$ and polyclonal anti-ER- $\beta$ reporter antibody labeled with Alexa Fluor 633 dye. They characterized the system by adding ER- $\beta$ into the solution. The loss in PL of the quantum dot was observed upon formation of the sandwich. The FRET distance between A and D was calculated to be $8-9 \mathrm{~nm}$, which is a proof of the larger size of the molecules [52]. Detection of the FRET signal between an antigen and an antibody can be improved using a specific separation approach, which was achieved through a capillary electrophoresis. In their study, $\mathrm{Li}$ et al. accomplished the labeling of mouse IgG and goat anti-mouse IgG with water-soluble CdTe QDs emitting at $532 \mathrm{~nm}$ (donor) and $632 \mathrm{~nm}$ (acceptor). FRET process was observed by separating noninteracting and well interacting FRET pairs using CE equipped with a fluorescence detector [53]. In contrast to this approach, the competition between a phage display-selected anti-TNT (2,4,6-trinitrotoluene) single-chain Fv fragment attached to CdSe/ZnS core/shell QDs (emitting at 530, 555, and $570 \mathrm{~nm}$ ) and a quencher dye (Black Hole Quencher-10)-labeled TNT analogue (TNB) was used to yield FRET-based quenching. As shown in Fig. 14.1, upon binding of TNT existing in the sample to the $\mathrm{scFv}$ fragment around the quenched quantum dot bound to TNB-BHQ-10, TNB-BHQ-10 was exchanged with TNT, which allowed a concentration-based increase in the quantum dot emission after termination of the quenching via FRET process [54].

Proteomic applications of antibodies are of great interest for detection of many diseases. Autoimmune diseases can be characterized using antibody profiling of the 


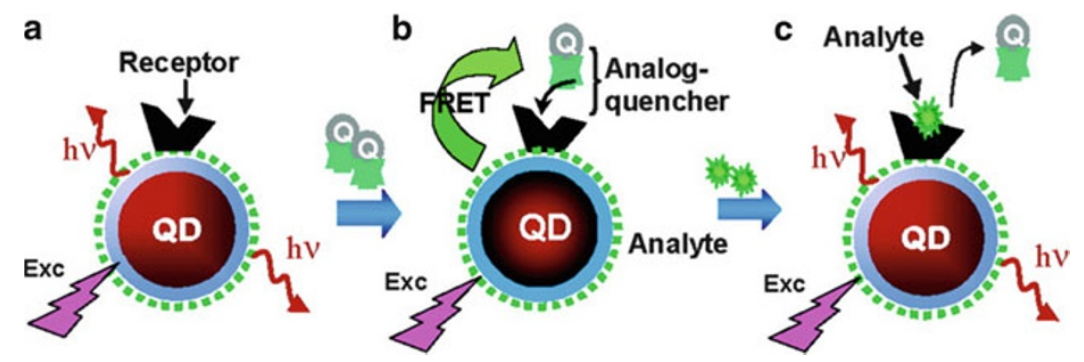

Fig. 14.1 Schematic of the TNT sensor (a) QD conjugate of the TNT receptor, (b) FRET between QD and quencher-labeled analogue molecule, and (c) replacement of the quencher-labeled analogue and recovering the QD fluorescent emission (Reprinted with permission from Goldman, E. R. et al. A hybrid quantum dot-antibody fragment fluorescence resonance energy transfer-based TNT sensor. J. Am. Chem. Soc. 127, 6744-6751 (2005). Copyright (2005) American Chemical Society)

disease markers, which is crucial for the diagnosis of the disease at early stages. In this manner, Sukhanova et al. demonstrated a sandwich-type assay by using the FRET effect enabled between orange emitting quantum dot nanocrystals and FITC dye. Quantum dots were assembled on microbeads and covered with a human DNA topoisomerase I (topol), which was used to detect anti-topol antibodies as they were one of the markers of systemic sclerosis. After the addition of the human plasma followed by the addition of FITC-labeled secondary anti-topol antibodies, the readout gave a FRET signal upon the existence of any anti-topol antibody in the human blood plasma [55].

\subsubsection{Monitoring Nucleic Acid Assembly and Functionality Using Quantum Dot Nanocrystal-Enabled FRET Processes}

Conjugation of the nucleic acids with quantum dot nanocrystals is a popular approach to build biosensors. Using the FRET process, the quantum dot nanocrystal-nucleic acid conjugates were used to detect the existence of certain nucleic acid sequences via sequence-controlled hybridization. Also, nucleic acid aptamers were utilized in this type of applications, which have specific recognition capabilities toward a specific molecule (e.g., protein, peptide, and DNA) via their corresponding three-dimensional structure. Compared to the dye-labeled proteinquantum dot conjugates, the dye-labeled thiolated DNA-quantum dot configuration makes more sensitive FRET sensors [56].

Molecular beacons are the well-known examples for the quantum dot-nucleic acid conjugations facilitating FRET process. In a molecular beacon, a singlestranded DNA (ssDNA) molecule is conjugated to a quantum dot nanocrystal from one end, and a quencher molecule is attached to the other end. The ssDNA molecule is generally designed in a way that can hybridize to forming a stem loop at the end. Generally, this stem-loop sequence contains a specific sequence that targets another specific region. Upon hybridization of the stem-loop sequence with the target probe, the stem loop opens and the distance between quantum dot nanocrystal 
Fig. 14.2 Schematics of a molecular beacon and its working principle a

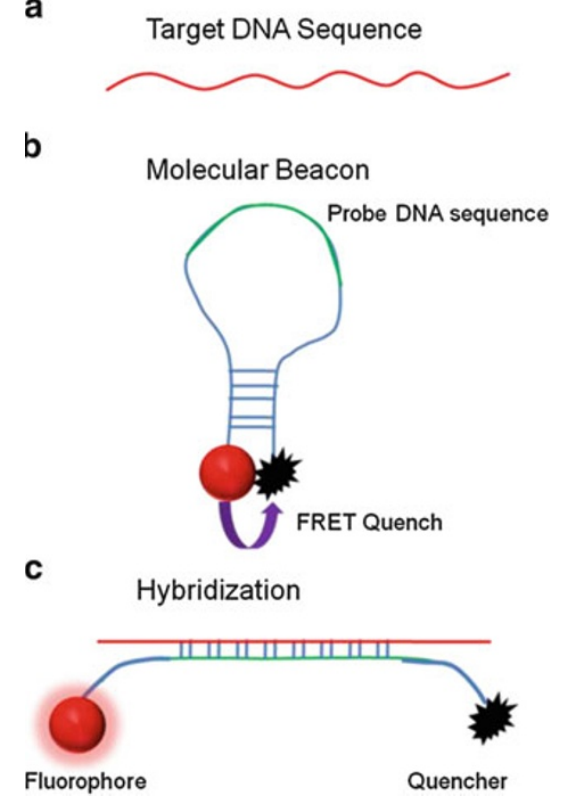

and quencher molecule increases, turning on the emission of the quantum dot upon the decrease in the FRET efficiency of the quantum dot with the quencher. Cady et al. studied the optimum conditions of molecular beacon configuration using quantum dot nanocrystal as the fluorophore (Fig. 14.2).

The attachment chemistry of quantum dot nanocrystal to ssDNA is crucial for the molecular beacon. Although streptavidin-biotin-based attachment is easy, using a zero-length linker, EDC chemistry is promising and provides a better fluorescence intensity. Depending on the emission wavelength of the quantum dot, using a dye molecule as a quencher is more promising instead of using gold nanoparticles, although it is a good quencher. However, the spectral overlap with the dye must be well-defined [57]. Wu et al. demonstrated the importance of the linker to conjugate DNA molecule to quantum dot after surface treatments for FRET-based sensing applications on a glass surface. Amine-functionalized DNA was attached to the carboxyl-containing quantum dots using NHS/EDC chemistry. Using a zero-length linker, they avoided any possible steric hindrance due to the size of the conjugates on the glass slide surface, where they achieved a $3 \mathrm{~nm}$ quantum dot conjugate in size and 70\% FRET efficiency during DNA detection via specific DNA hybridization of the target DNA molecule with a surface-bound reporter [58]. Similarly, using thiols is also very useful as a linker for quantum dot-DNA conjugates in FRET applications [56]. Quantum dot molecular beacons were also formed using quantum dot nanocrystals emitting at different wavelengths, which brought about a promising approach for the multiplexed quantum dot molecular beacon systems. In a multicolor quantum dot nanocrystal scheme, molecular beacons were able to detect a target DNA down to $8 \mathrm{ng}$ in a sample solution, which was comparable to 
that of the currently available organic dyes with a higher stability and a lesser background signal [59].

Single quantum dot-based DNA nanosensors have been very popular since the demonstration of its first usage. In this scheme, a dye molecule was attached to quantum dot nanocrystals by using appropriate conjugation chemistry. Cy5 and quantum dot nanocrystals emitting at 605 were found to have an excellent overlap, and in most of the following studies, this pair was used for FRET-enabled sensing. In the demonstration by Zhang et al., this configuration was found to be more sensitive than a molecular beacon approach [60].

DNA aptamers are molecular recognition elements functioning through their three-dimensional structures. They were selected using a system called SELEX. Using an aptamer molecule selected for adenosine triphosphate (ATP) binding, Chen et al. built quantum dot nanocrystal-enabled FRET-based systems to sense ATP in solution, which has an alternative configuration. In the nanosensor, a streptavidin-coated quantum dot nanocrystal $(605 \mathrm{~nm})$, a 3'-biotin-modified DNA, 3'-Cy5-labeled DNA, and a capture DNA involving an ATP-binding aptamer that contains specific binding sequences for $3^{\prime}$-biotin-modified DNA were used. In the absence of ATP, the capture DNA was bound to the $3^{\prime}$-Cy5-labeled DNA and 3'-biotin-modified DNA, which later was bound to the streptavidin-coated quantum dot nanocrystal complex via biotin. In this first configuration, the bound $3^{\prime}$-Cy5labeled DNA came into the close proximity with the quantum dot, resulting in the quenching of the quantum dot due to the FRET process. However, if ATP was introduced, it competed with the $3^{\prime}$-Cy5-labeled DNA and released it. This resulted in an obvious increase of the quantum dot emission [61].

This alternative approach might be extended into sensing DNA-DNA, DNA-protein, and DNA-small molecule interactions (e.g., minerals and vitamins). Moreover, a nanosensor for cocaine detection was realized using a similar approach based on a DNA aptamer specific for cocaine. This sensor, however, was built in two different configurations. A quantum dot nanocrystal emitting at $605 \mathrm{~nm}$ was conjugated with a DNA molecule displaying a cocaine-specific aptamer sequence and could also hybridize with a DNA fragment conjugated with Cy5. In the absence of the cocaine, the Cy5-conjugated DNA fragment was bound to the quantum dot nanocrystal conjugated backbone, and the FRET process between Cy5 and quantum dot nanocrystal causes the quantum dots to be quenched. Upon the addition of cocaine into the medium, it was bound to the aptamer sequence which has caused the dissociation of the Cy5-labeled DNA fragment and triggered the increase of quantum dot PL upon turning down the FRET process. Furthermore, the same process was improved by introducing another dye-labeled (Iowa Black RQ) DNA fragment. This secondary fragment (Iowa Black RQ) DNA can bound to the DNA attached to the quantum dot nanocrystal and formed a sandwich shown as in Fig. 14.3 in the absence of cocaine which resulted in quenching the emission of Cy5. In the presence of cocaine, the aptamer was bound to cocaine, which resulted in the release of Iowa Black RQ-labeled DNA. After this, the fluorescence of Cy5 became detectable as a sign of cocaine [62]. 


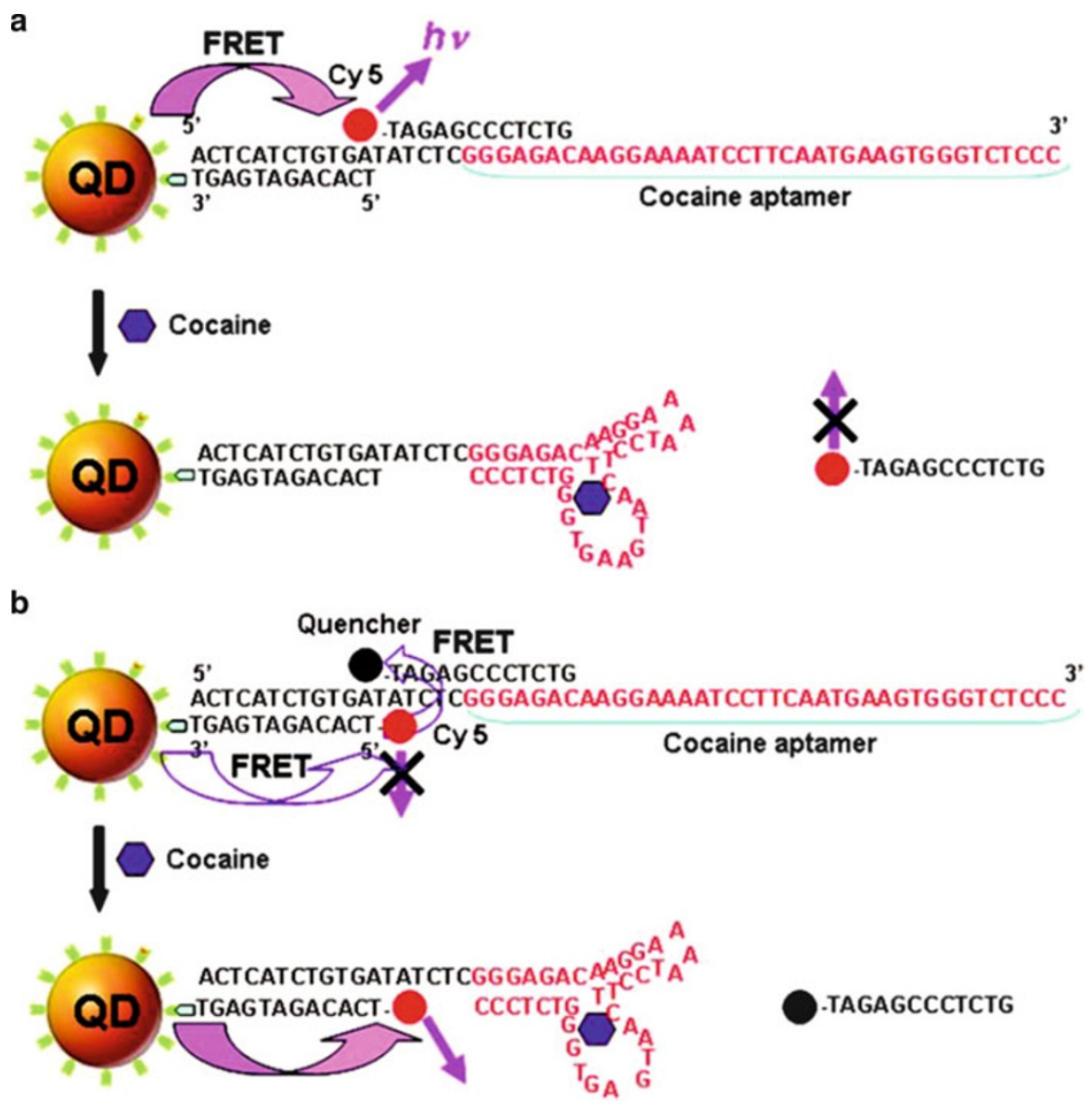

Fig. 14.3 Schematic of the cocaine sensor: (a) Signal-off configuration of the QD-based cocaine sensor, where the QD emission was quenched at first and later increased upon cocaine binding. (b) Signal-on configuration of the QD-based cocaine sensor, where the QD emission was quenched upon cocaine binding (Reprinted with permission from Johnson, L. W. \& Zhang, C. Y. Single Quantum-Dot-Based Aptameric Nanosensor for Cocaine. Anal Chem 81, 3051-3055, doi: 10.1021/Ac802737b (2009). Copyright (2009) American Chemical Society)

Detection of the platelet-derived growth factor (PDGF) using an aptamer DNA conjugated with quantum dot nanocrystals was also realized, where the FRET was switched off and on by the attachment and release of the black hole quencher to the quantum dot-conjugated DNA sequence upon the absence and existence of PDGF in the sample [63]. Alternatively, unlabeled DNA can be also captured to its specific capture agent, and detection of target DNA can be achieved. In this configuration, the capture DNA was conjugated to a quantum dot nanocrystal using polyethylene glycol (PGE) linker. PEG linker helped to adjust the distance of capture DNA to quantum dot nanocrystal, which helped to adjust FRET distance between quantum 
dot nanocrystal and dye, ethidium bromide (EtBr), a molecule labeled to target DNA. To detect the unlabeled DNA, the sample was introduced into the solution and the hybridization took place. Later, EtBr was added to the solution and it formed a fluorescent complex with unlabeled DNA. Upon the formation of the complex, FRET occurred between the quantum dot nanocrystal and the complex, which gave a rise in the fluorescent emission of ethidium bromide [64]. In another innovative approach, it was proposed to detect the DNA hybridization using a twostep FRET system. In this route, a positively charged conjugated polymer was used as the light-harvesting antenna in the solution. It was bound to negatively charged CdTe QDs, upon which FRET process took place and the outer surface of QD-antenna complex became positively charged. This complex was then interacted with the labeled DNA fragments in solution. The second FRET process took place between the dye-labeled DNA molecule and quantum dot-conjugated polymer complex. It was also noted that ssDNA and double-stranded DNA (dsDNA) created different slopes in linearly increasing ratio of dye to quantum dot nanocrystal emission intensities because of the difference in their binding strength to QD-polymer complex [65].

A tumor marker, mucin 1, is a glycoprotein that was expressed on most epithelial cells. This marker was known to be a good indicator for early cancer diagnosis. A nanosensor that involved a specific DNA aptamer for a MUC1 was constructed, where the DNA aptamer was bound to the quantum dot through EDC/NHS chemistry. The quencher ssDNA, which involved the Iowa Black quencher dye, was able to hybridize with the MUC1 DNA aptamer; however, in the presence of MUC1, the aptamer was unfolded into ssDNA. Therefore, both the quencher ssDNA and quantum dot-modified ssDNA were able to attach on the aptamer. This hybridization brought the quencher DNA and the quantum dot in close proximity resulting in FRET-enabled quenching of the quantum dot, which was shown to be MUC1 concentration dependent [66].

Besides using chemical conjugation or streptavidin-biotin conjugation, for the assembly of ssDNA to quantum dot nanocrystals, the electrostatic interaction was also employed to build FRET facilitating system to probe micrococcal nuclease activity detection. In this configuration, $\mathrm{CdSe} / \mathrm{ZnS}$ nanoparticles were functionalized with an enzyme, lysozyme, to prepare positively charged quantum dots to assemble a negatively charged dye-labeled ssDNA. The dye-labeled ssDNA was a substrate for nuclease activity on the outer surface of the quantum dot nanocrystal. When the complex was brought in contact with the micrococcal nuclease, the nuclease digested the ssDNA and caused the release of the dye molecule. This process was monitored as a decrease in the emission of the dye molecule upon deterioration of the FRET process through nuclease activity [33].

To detect the target DNA more precisely, a DNA binding and cleaving enzyme, nicking endonuclease, was utilized by $\mathrm{Xu}$ et al. In the assay, they used a linker DNA, which was fully complementary to the target DNA. First, the target DNA and the linker DNA were brought together and they formed a dsDNA, which had a site for the endonuclease to recognize. The endonuclease cleaved dsDNA and the target DNA was released, and this recycle was repeated for many times by resulting in the 
cleavage of many linker DNAs. In the second stage, first the enzyme was denatured, and then the quantum dot-conjugated probe I DNA and rhodamine B-conjugated probe II DNA were added, while the uncleaved linker DNA was hybridized with the quantum dot nanocrystal-probe I. Subsequently, the hybrid was sandwiched with rhodamine B-probe II and led to the FRET process. Because the ssDNA target can be used for many cycles, this assay was found to be capable of DNA detection down to $1 \mathrm{nM}$ level [67].

In addition, for the detection of the target DNA molecules in solution, also the DNA methylation was probed using a quantum dot-based FRET process in another approach, which is an important tool to detect cancer and monitor tumor behavior for targeted therapy. For this purpose, a sensitive and fast methodology was developed and the established method was called MS-qFRET. In this approach, the genomic DNA was extracted from the sample and subjected to sodium bisulfite conversion. First, the reaction converted the unmethylated cytosines to uracil and the methylated ones were not affected. Second, the sample genomic DNA was amplified with polymerase chain reaction (PCR) using biotin and organic redemitting fluorophore labeled forward and reverse primers. Finally, the streptavidin-coated nanocrystal was assembled with the biotin and dye-tagged sample DNA. During the PCR reaction, elongation was interrupted by uracils (converted from unmethylated cytosines). Thus, the higher ratio of methylation led to the longer DNA sequence and increased the number of dye molecules that were attached. Consequently, the methylation ratio was determined from the quenching of quantum dot nanocrystals upon FRET with the dye molecules within the close proximity [68]. Suzuki et al. also demonstrated a multipurpose approach using DNA-conjugated quantum dot nanocrystal system for FRET process, which aimed at responding to nucleolytic cleavage, DNA polymerization, and $\mathrm{pH}$ change. DNA digestion was monitored upon specific cleavage of the DNA molecule by a nuclease. However, depending on the construction strategy of the DNA-quantum dot nanocrystal conjugates, two distinct FRET patterns were observed. In DNA elongation experiment, upon addition of the Klenow fragments and their elongation with dye-labeled dUTPs, the increased fluorescence of the quantum dot nanocrystals was observed. By the attachment of fluorescein dye to the aminefunctionalized quantum dot nanocrystals using Traut's reagent, a FRET-based pH-sensitive sensor was constructed [69].

A nanocrystal-based FRET sensor has also been constructed for the detection of RNA. In their work, Bakalova et al. demonstrated a siRNA sensor to test the effectiveness and activity of the siRNA fragments with the aim of using them in RNA interference applications in mammalian cells. For that, they conjugated their designed siRNA sequence with a cross-linker to the carboxyl-coated quantum dot. They amplified the mRNA extracted from the target sample and labeled Cy5-labeled nucleotides, while the mRNA sequence was amplified. Cy5-labeled mRNA was used as a reporter during the hybridization with the siRNA-modified quantum dots. If the siRNA fragment is a good candidate for the target mRNA and hybridizes with the siRNA conjugated to quantum dot nanocrystals, then a FRET process took place between Cy5 on mRNA and the quantum dot nanocrystal 
conjugated to siRNA. This led to an increase in the emission of Cy5. However, if the increase in the emission of Cy5 was limited or cannot be detected, then a mismatch was observed instead of the hybridization. Compared to the available techniques, this approach is faster and promising as a route for siRNA screening [70].

Another similar experiment was carried out to test the drug candidates for AIDS, where the sequence-specific interaction between regulatory protein Rev of HIV virus and portion of env gene within RNA gene (Rev-responsive element, RRE) was targeted. Since this interaction is vital for the HIV-1 replication, any attempt to destroy this interaction may lead to the discovery of new drugs for HIV. With this motivation, Zhang et al. proposed a sensor, which probes the interaction between the RRE RNA and a 17-mer peptide derived from Rev. In this configuration, RRE RNA was labeled with a biotin and later conjugated to the streptavidin-coated quantum dot emitting at $605 \mathrm{~nm}$. Rev peptide was modified with Cy5 from its N-terminus. FRET process took place when the Cy5-Rev peptide was bound to the QD-RRE RNA, with an increase in Cy5 emission. The inhibitory effect of the proflavin was tested within the system, where the proflavin was expected to dissociate the Cy5-Rev peptide and decrease the Cy5 emission upon corruption of the FRET process. However, due to the long red tail of proflavin emission, it was not possible to see the decrease in emission of Cy5, only the expected increase in the quantum dot emission was detected. Using correct dichroic and band-pass filters, the leaking long red tail of proflavin emission was filtered, and a distinct suppressed emission from Cy5 was obtained when proflavin triggered the dissociation of Cy5-Rev peptide from RRE RNA-conjugated nanocrystals [71].

Nanocrystal-based FRET research was carried out in solution as the ensemble measurements. However, it was found that it is possible to enhance FRET signal between the donor quantum dot and the acceptor dye-attached DNA by using capillary electrophoresis system. This enhancement phenomenon was observed because of the deformation of the DNA molecule in the capillary flow [72]. Therefore, it can be useful to search for the methodologies other than these ensemble measurements to enhance the FRET signal, which is possibly as important as the conjugation strategies and linkers to be utilized.

\subsubsection{Monitoring Protein Involved FRET Processes}

Many applications of biosensors in the literature heavily depend on the utilization of proteins as the recognition elements of the biosensor of interest [73]. They have also been utilized in many different FRET applications, and dye-conjugated proteins served as the acceptor or donor molecules. In some of these studies, the proteins were used without any dye conjugation as they already have a selffluorescence originated from amino acids such as tryptophan, tyrosine, and phenylalanine $[74,75]$. Sarkar et al. have showed this phenomenon by conjugating human serum albumin (HAS) to a CdS quantum dot emitting at $510 \mathrm{~nm}$. In this configuration, when HAS was used as a donor and the quantum dot was as an acceptor, FRET was facilitated between the tryptophan at the position of Trp214 and the quantum dot. HSA was conjugated to CdS nanocrystal, and upon thermal 
folding/unfolding, which basically modulated the distance between the quantum dot and Trp214, the steady state PL and time-resolved PL were monitored so that a structural nanosensor was introduced to track protein-folding intermediates [76]. Since FRET signal occurred at distances lower than $10 \mathrm{~nm}$, it is possible to track the changes in the conformation of a given protein labeled at appropriate regions with proper acceptor-donor dye choices. Depending on the conformation of the protein, the distance between the labeled regions may facilitate FRET, if the conformation of the protein is changed, and the distance and hence the FRET signal between the labeled residues change, too. The FRET signal being a nanoruler for the molecular distances can thus act as a probe for tracking the changes in the protein structure.

The phenomenon of using the structural changes as a probe in conjugation with an eventual change in the FRET signal was also tested using a model protein, maltose-binding protein (MBP). Hybrid assemblies of the maltose-binding protein and quantum dots were shown to be a model biosensor. Maltose-binding protein from Escherichia coli was attached to quantum dots emitting at $560 \mathrm{~nm}$ via His-tag expressed at the $\mathrm{C}$-terminus of the protein. Later, the MBP-quantum dot assembly was brought in contact with a dye-labeled maltose analogue molecule, $\beta$-cyclodextrin-QSY9 (dark quencher). Then $\beta$-cyclodextrin-QSY9 was bound to the saccharide-binding site of MBP. Upon binding of the $\beta$-cyclodextrin-QSY9, the quantum dot emission was quenched because of the FRET between $\beta$-cyclodextrinQSY9 and the quantum dot. When maltose molecules were added to the solution, the cyclodextrin-QSY9 dissociated and the maltose was bound to the MBP maltosebinding site, which gave rise to a signal increase in the quantum dot emission by virtue of the breakdown of the FRET-based quenching. Consequently, the maltose concentration-dependent increase in the quantum dot emission was observed. Utilizing the distance dependence nature of FRET in this configuration, another approach was put forth. In this technique, possible problems derived from the donor-acceptor distance restrain can be overcome by the utilization of a multiple step FRET process. In the second configuration, a two-step FRET process was employed where MBP was both conjugated to the quantum dot emitting at $530 \mathrm{~nm}$ as the donor and a $\mathrm{Cy} 3$ dye as the acceptor emitting at $570 \mathrm{~nm}$. First, the FRET process took place from the quantum dot to the $\mathrm{Cy} 3$ dye, and the second FRET process occurred between the $\mathrm{Cy} 3$ and the $\beta$-cyclodextrin-Cy3.5. The second FRET process resulted in the quenching of the Cy3. Again, addition of maltose molecules replaced the $\beta$-cyclodextrin-Cy3.5, and removal of this molecule was detected as an increase in the emission of $\mathrm{Cy} 3$ in a maltose concentration-dependent manner. This system was well studied from different aspects including the concentration dependency and the utilization of different donor and acceptor molecules for optimal functioning [77-79].

Detection of the enzymatic activity by manipulating the FRET process between the quantum dot and an acceptor molecule was also demonstrated as biosensors. Protease activity has been one of the most detected phenomena in the biosensor literature. Also, protease sensors were proposed and implemented using quantum dot-enabled FRET processes. Chang et al. proposed a structure where gold nanoparticles (AuNPs) were conjugated with the quantum dot nanocrystal by 
exploiting a peptide linker (GGLGPAGGCG), which was a proper target for a protease called collagenase XI. Decoration of the surface of the quantum dot with AuNPs quenched the optical emission. Protease was added into the solution, and the peptide linkers were digested by collagenase, and the AuNPs were released from the close proximity of the quantum dots, which led to the increased QD emission [80]. A similar strategy was also realized to detect type IV collagenase, which finds applications in tumor invasion and metastasis [81]. In a mechanistic study, nonradiative quenching of the quantum dots due to the proximal gold nanoparticles was investigated by means of distance control between the quantum dots and AuNPs using a peptide sequence (YEHK), which was recognized to exhibit a rodlike structure, with its second structure enabling a rigid nature. In this approach, the chain length was changed, and it was concluded that the quantum dot quenching arose due to the long-distance dipole-metal interaction [82].

Attachment of a dye molecule to a quantum dot is one of the most popular approaches, which were also explained in the previous section. Similarly, various peptide sequences were employed as mentioned above. For example, Shi et al. followed a similar approach and functionalized the surface of quantum dots with a short peptide (RGDC) terminated with rhodamine Red-X, which quenched the quantum dot emission in the close proximity. Upon addition of collagenase and subsequent cleavage of the peptide, FRET was ceased, consequently increasing the quantum dot emission and quenching of the rhodamine Red-X [83]. Using the same quantum dot-RGDC-rhodamine Red-X, the effect of the inhibitory activity of various chemical agents was tested in vitro, where the inhibition of the enzymatic activity of trypsin was tracked [84]. Clostridium botulinum is attributed to be one of the well-known pathogens and is being characterized by the extracellular production of a toxin called botulinum, composed of a light and heavy chain dimer and activated just after the posttranslational modifications. Posttranslational modifications through proteolysis produce the chains by breaking disulfide bond between them. Sapsford et al. proposed a quantum dot nanosensor to detect the activity, thus the presence of the toxin. The light chain of the toxin (LcA) was used as the target molecule, which showed an endopeptidase activity. His-tagged peptide targets were prepared into which an LcA binding side and a helix spacer were incorporated, which were labeled with a Cy3 and resultantly caused the quenching of quantum dots. Sapsford et al. utilized two approaches. The first was the popular metal affinity conjugation of the peptide to the quantum dot, and they assayed the LcA activity through the increase in the PL emission of the quantum dots upon detachment of the Cy3-containing part of the peptide. They also proposed another approach in which the LcA protein and the substrate peptides containing Cy3 and His-tag were mixed. Upon proteolytic activity of LcA, the peptide was broken down into parts. Therefore, upon addition of the QD, undigested peptides were stuck on them. This attachment caused decrease in the PL emission and read out as the signal for the LcA activity when compared to the initial peptide concentration [85].

Caspase is an important protease with its particular relevance to cancer research and one of the key signs of apoptosis. Boeneman et al. constructed a nanosensor to detect caspase- 3 activity in vitro. A cleavage site for caspase- 3 protease activities 
was incorporated into a fluorescent protein mCherry through protein engineering, which was terminated with His-tag sequence to attach the protein to the quantum dot. The kinetics of FRET process was monitored as a function of proteolytic activity of caspase- 3 in real time so that the Michaelis-Menten descriptors for caspase-3 were extracted as well [40]. Similar to this approach, Prasuhn et al. demonstrated a caspase- 3 sensor by utilizing a chemical dye, Texas Red, as the acceptor molecule [86]. Biswas et al. designed and implemented an alternative protease sensor for in vivo applications. In this design, elastine-like peptide (ELP) and TAT sequence were also incorporated in addition to the protease cleavage site, dye molecules, and quantum dot. The penetration of the quantum dot was carried out using the ELP peptide and TAT sequence. The quantum dots were conjugated with an organic dye, Alexa 568, through a cysteine group contained by a peptide expressing protease cleavage site and attached to ELP and TAT. The quantum dot conjugate cargo was delivered into the HeLa cells and monitored under a fluorescence microscopy. Later, the cells were transfected with a proviral plasmid pNL4-3.HSA.R-E- to induce the production of noninfectious HIV viruses. Following the delivery of the quantum dot probe, the cleavage of the peptide with the HIV-1 Pr was monitored as a decrease in the intensity of Alexa dye. Inhibition of HIV-1 Pr upon addition of ritonavir, saquinavir, and indinavir was detected as an increase in the FRET efficiency in a dose-dependent manner [87].

Kinase-enabled protein phosphorylation is recognized as one of the key cellular processes any dysfunction of the kinases has been related to in the development of a disease state, and kinases are important for cell signaling and cancer biology. Ghadiali et al. exploited quantum dot nanocrystals attached with reporter peptides involving serine, tyrosine, and threonine amino acids, where the attachment was achieved through using His-tagged peptides. In the existence of kinase, the corresponding amino acids were phosphorylated, and FRET partner dye-labeled anti-phospho-amino acid antibody was brought in contact with the quantum dotpeptide conjugate. While the anti-phospho-amino acid antibody was bound to the phosphorylated peptides, the quantum dot emitting at 605 and Alexa Fluor emitting at 670 were brought in close proximity satisfying the FRET conditions [88]. The same group also constructed another sensor to monitor the activity of the histone acetyltransferase (p300 HAT). They utilized the same approach for the kinase sensor and synthesized a target peptide mimicking $\mathrm{H} 4 \mathrm{~N}$ terminal histone sequence including His-tag. The peptide sequence was first incubated with the p300 HAT and acetylated at the Lys $_{6}$ position. Later, dye-labeled acetyl lysine specific antibody recognized the target peptide and was bound to it, causing a FRET-based quenching of the quantum dot, which was monitored as a sign of HAT activity [89].

Being responsible for the $\beta$-lactam antibiotic resistance in bacteria, detection of $\beta$-lactamase is crucial for clinical samples. In their work, $\mathrm{Xu}$ et al. proposed a quantum dot-enabled assay method, which relied on a signal-off type of FRET interaction. In this approach, $\mathrm{CdSe} / \mathrm{ZnS}$ quantum dot nanocrystals were decorated with streptavidin, and the Cy5 dye-conjugated and biotin-modified $\beta$-lactam molecules were synthesized. To eliminate steric hindrance, which arose due to the deep binding pocket of streptavidin (SA), a linker was also introduced to the $\beta$-lactam 
molecules after the biotin modification. After the conjugation of quantum dot-SA with biotinylated linker- $\beta$-lactam-Cy5 complex, the quantum dot emission was observed to quench indicating the formation of the nanosensor. Meanwhile, the lactamase was added into the nanosensor solution and, due to the degradation of $\beta$-lactam, Cy5 was released into the solution and diminished the FRET process, which in turn resulted in an increase in the quantum dot emission (by fourfolds in this case) [90].

Lipid-coated quantum dots have been developed to be utilized as a probe for lipid exchange at the cellular interface. In order to understand the capability of the lipid-coated quantum dot nanocrystals for this purpose, a quantum dot-based architecture that facilitated FRET process was proposed. This lipid-coated quantum dot structure was composed of a quantum dot coated with DMPE, a phospholipid that displayed an available amine group. Through the further modification of the amine group, an infrared dye Cys5.5 was conjugated to the quantum dot surface. For a second nanoparticle conjugate to mimic a natural high-density lipoprotein (HDL), the particle was further incubated with apolipoprotein (apoA $\sim 1$ ). First, the QD-HDL without Cy5.5 was tested for the lipid exchange ability with the Cy5.5-HDL and Cy5.5-micelles. These two particles were mixed, and it was observed that because of the exchange of the lipids, Cy5.5 was also transferred to the QD-HDL structure, which was observed as a decrease in the emission of the QDs due to the FRET process between the QD and the Cy5.5 dye. The effectiveness of the Cy5.5-labeled QD-HDL particles was tested for their functionality by incubating them with living j774a.1 macrophage cells. Upon the adhesion of the Cy5.5-labeled QD-HDL nanoparticles, Cy5.5-lipid exchange with the cell membrane caused the FRET to disappear. This process was observed in the course of transferring the quantum dot conjugates from the cell membrane into the cellular organelles [91]. The schematic of the approach was displayed in Fig. 14.4.

Rakovich et al. realized a FRET facilitating system to increase the lightharvesting efficiency of the bacteriorhodopsin (bR) embedded into the purple membranes (PM) of a bacteria Halobacterium salinarum. Quantum dot nanoparticles conjugated with PM were tested for their effectiveness by monitoring the FRET-based quenching of the quantum dots. The formation of the FRETprocessing QD-PM assembly increased the light-driven transmembrane pumping by enhancing light-harvesting capability of bR [92] (Fig. 14.5).

Seker et al. proposed and constructed a film-based biosensing approach where the quantum dots emitting at $640 \mathrm{~nm}$ were used as the acceptor and the quantum dots emitting at 560 were used as the donor for FRET process. Negatively charged CdTe quantum dots were layer by layer assembled using polyelectrolytic peptides. The thermodynamics of the assembly process of the peptides was investigated as well as the structural features of the peptides to form the most reliable film combination. Supramolecular assembly of the peptide pairs with different chain lengths was tested for most reliable design of the FRET facilitating quantum dot nanocomposites. Once the best peptide constructs were realized, control of the FRET efficiency was achieved using trypsin enzyme. Trypsin enzyme cleaved specifically the linker peptides, which triggered the destruction of peptidic thin 


\section{a}
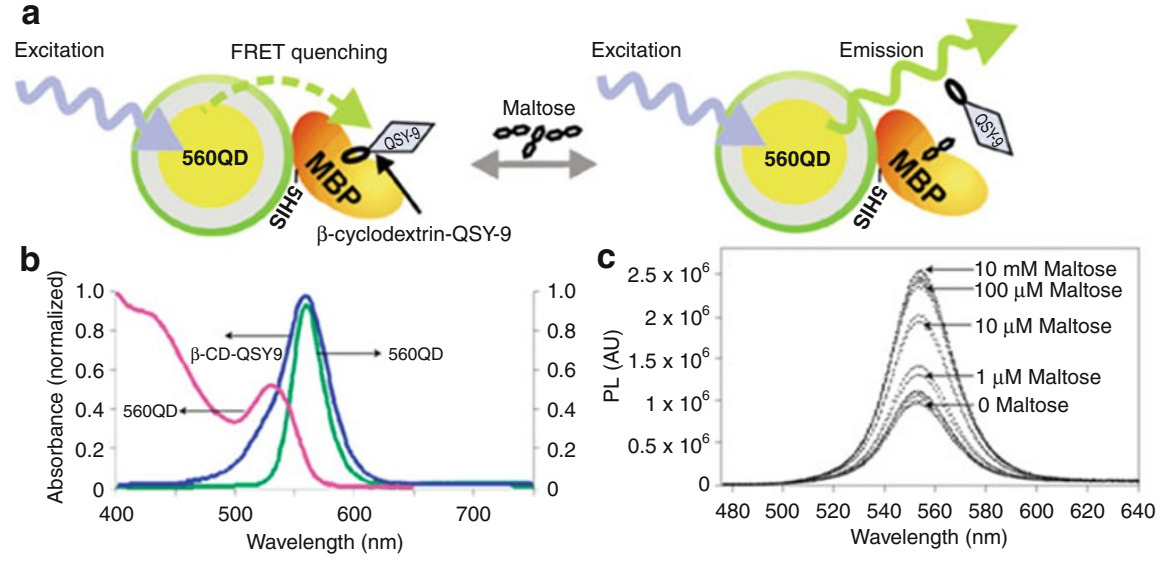

Fig. 14.4 (a) QD-FRET sensor schematics, (b) spectral properties of the sensor: absorption (pink) and emission spectra (green) of MBP-conjugated 560QD, along with the absorption spectra (blue) of beta-CD-QSY9. (c) 560QD-10MBP maltose sensing (Reprinted by permission from Macmillan Publishers Ltd: [Nature Materials] (79), copyright (2003))

a

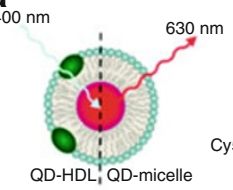

b

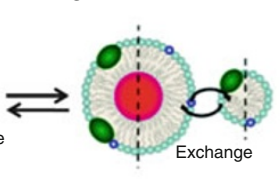

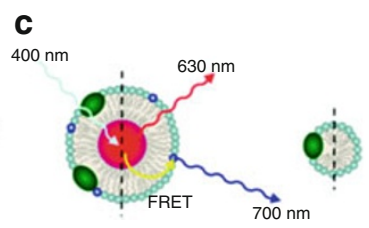

Fig. 14.5 The schematic of FRET facilitating lipid-coated quantum dots: (a) HDL-coated QD and QD micelle with Cy5.5-HDL and Cy5.5-micelle without FRET, (b) Cy5.5 dye exchange between the HDL-QD and HDL-micelle, and (c) FRET between the Cy5.5 and QD in both QD-micelle and QD-HDL configuration (Reprinted with permission from Skajaa, T. et al. Quantum Dot and Cy5.5 Labeled Nanoparticles to Investigate Lipoprotein Biointeractions via Forster Resonance Energy Transfer. Nano Lett 10, 5131-5138 (2010). Copyright (2010) American Chemical Society)

film. In the study, instead of the PL intensities of the donor and acceptor quantum dots, the change in the fluorescence lifetime of these QDs was used as the readout signal. Following the release of the donor quantum dots from the peptidic film, the remaining acceptor quantum dots exhibited a substantially decreased lifetime [93] (Fig. 14.6).

\subsection{Quantum Dot Nanocrystals Enhanced Bioimaging}

Quantum dot-based FRET processes were incorporated in many different biosensing designs, some of which were discussed above. In some of these applications, the quantum dot-based FRET sensors were also delivered into the cells 


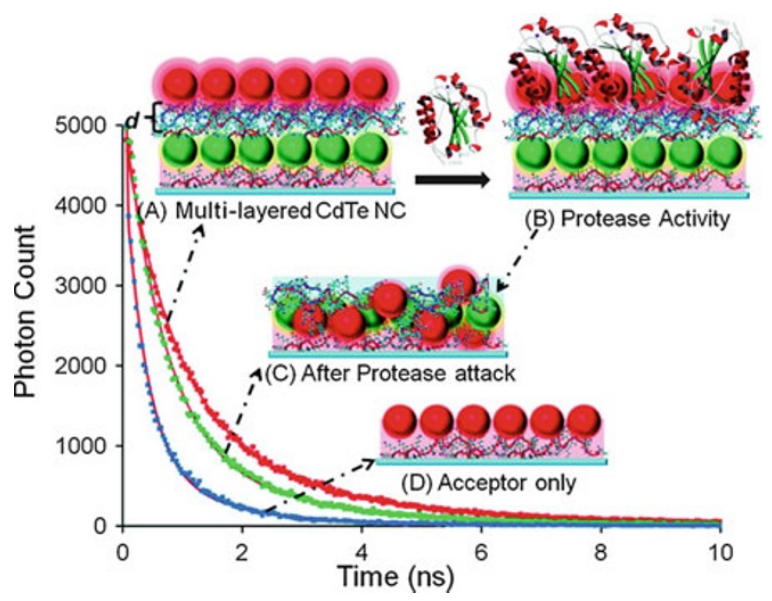

Fig. 14.6 Peptidic FRET facilitating nanocomposite film: (a) PL decay of the multilayered structure facilitating FRET, (b) the proteolyic digestion of the peptide film, and (c) PL decay following the rearrangement of the peptide film as a result of the proteolyic digestion, and (d) PL decay of the acceptor alone (Reprinted with permission from Seker, U. O. S. et al., Peptide-Mediated Constructs of Quantum Dot Nanocomposites for Enzymatic Control of Nonradiative Energy Transfer. Nano Letters 11, 1530-1539 (2011). Copyright (2011) American Chemical Society)

to monitor any specific cellular process. For imaging applications, the same approach for constructing biosensors was followed, and the formed constructs of the quantum dots were delivered into the cells to track cellular function or to deliver biomolecules or drugs, which were later monitored by the FRET signal in real time.

Shaheen et al. utilized the FRET process involving quantum dots to monitor the state of the condensation and decondensation of the plasmid DNA (pDNA). pDNA was attached to the quantum dot nanocrystal, and FRET was achieved between this conjugate and rhodamine-labeled polycations. The pDNA-polycation core was then encapsulated into a lipid vehicle, which was called the tetra-lamellar multifunctional envelope-type nanodevice (T-MEND) to transfect HeLa cells. Following the penetration of the loaded T-MEND into the cell, the release of the pDNA was tracked by imaging the change in the FRET signal using a confocal microscope, which has also scanned the T-MEND device in z-stack to detect the condensation/decondensation of the pDNA embedded into the T-MEND device. This method brings about a new and feasible approach for the optimization of the gene delivery tools for gene therapy using the FRET signal as a guide [94].

Bagalkot et al. constituted a hybrid quantum dot system, which was conjugated with an A10 RNA aptamer, to recognize the extracellular domain of the prostatespecific membrane antigen (PSMA). Aptamer-QD conjugate was loaded with an anticancer drug named as doxorubicin. Studies showed that this system facilitates two distinct FRET processes. First, a FRET process occurred between the quantum dot and the doxorubicin molecule, where the quantum dot served as a donor. Second, a FRET process took place between the aptamer molecules and doxorubicin molecules where the doxorubicin acted as a donor. This multi-FRET system 
was first delivered into the PSMA-expressing prostate adenocarcinoma cell lines with the help of A10 RNA aptamer molecule through molecular recognition. After the delivery of the particle cargo, the doxorubicin was released into the cellular environment. Release of the doxorubicin reverted the PL emission of the quantum dot. Upon release of the doxorubicin, PL of doxorubicin was also recovered as it was dissociated from the A10 RNA aptamer. Therefore, both the delivery of the quantum dot composite and the release of the drug were monitored through imaging; additionally, the therapy of the cells was succeeded with the released drug [95].

The localization of the protein in living cells is important for the protein distribution. The available techniques can give some idea about the existence of these proteins but not about their interaction depending on their localization. As a solution, with the method Kang et al. proposed, it is possible to track the existence of neighboring proteins. In this work, nucleolin protein was labeled with a dyemodified aptamer molecule, AS1411 targeting nucleolin. On the other hand, integrin was marked with a quantum dot nanocrystal. To recognize the integrin, the quantum dot was decorated with an RGD sequence, which exhibits a high affinity toward integrin protein. Following the assembly of the quantum dot and dye molecules, a FRET process between the dye and the quantum dot was tracked. Using the microscopy analysis, the expression levels of integrin and nucleolin were determined. This approach allows to co-localize various proteins in a native cell, which are important to determine the function of some of the important genes [96]. Intracellular imaging of levels of a given material was also achieved using quantum dot-enhanced FRET, for which Wang et al. used a calcium-modulating protein (CaM)-QD sensor to track the intracellular $\mathrm{Ca}^{+2}$ level based on FRET [97].

\section{$6 \quad$ Conclusions and Future Perspective}

Quantum dot nanocrystals have been exploited in FRET applications for probing different biological processes. FRET-based labeling systems were designed typically using quantum nanocrystal as the donors, while the organic dye molecules or fluorescent proteins were utilized as the acceptors. Also nanoparticles like silica nanoparticles and gold nanoparticles were used as the acceptor species. To satisfy the FRET conditions, donors and acceptors were conjugated using different approaches. Most popularly, the quantum dots and acceptor species were conjugated using a chemical bonding approach, where the carboxyl-functionalized quantum dots were activated using NHS/EDC and then active amine-binding quantum dots were linked. Streptavidin-coated quantum dots were also commonly conjugated with the biotin-labeled acceptors, or as an alternative approach, the conjugation was obtained using electrostatic interaction between the quantum dot donors and dye acceptors.

Many assays were designed and realized for quantum dot-based FRET applications. Antibody-antigen interactions, protein-protein interactions, and enzymatic activities were successfully monitored using quantum dot-enabled FRET systems, 
where a pre-quenched quantum dot was typically used as the donor and an increase in the quantum dot PL was observed upon interaction of the targeted entities. Similarly, in the design of molecular beacons or DNA-hybridization assays, the same approach was used, which is commonly known as the signal-off mode FRET system. Additionally, another FRET mode, in which initially the quantum dot emission was not quenched and the acceptor was not in the close proximity of the donor or was quenched by a secondary acceptor due to its interaction with another molecule, the FRET conditions were later satisfied only upon the interaction with the targeted molecule.

Most of the previous studies in the literature focused on using the quantum dot nanocrystals as the donor molecules because of their long fluorescence lifetime, which makes the resulting FRET efficiency higher. Although there are challenges in using quantum dots as the acceptors, utilizing biological reactions (e.g., those based on chemoluminescence and bioluminescence) can enable the quantum dots to emit in the absence of a physical light. This kind of approaches can lead to the development of innovative bioassay systems.

Although there have been fascinating efforts put into action to improve the capabilities of the quantum dots, there are still challenges especially for the in vivo delivery of these dots. There have been numerous different approaches and tools developed and optimized for a simple delivery of the quantum dots. Utilization of lipid transfection, direct microinjection, and electroporation are among the widely studied approaches in this area [98-100]. The surface chemistry of the quantum dots affects the delivery in the cellular environment. For that reason, utilization of protein and peptide-integrated quantum dots is becoming more important. Biomolecules are generally recognized to be biocompatible compared to the chemical linkers. For example, signaling molecule-functionalized quantum dots can be used for the transportation of the quantum dots across the cellular membrane to the nucleus of the cells.

FRET-based approaches to track any given biochemical reaction occurring within the cell are capable of creating a new era for the proteomics. Given the fact that metabolic engineering needs real time monitoring of the metabolic flux analysis of a molecule of interest, quantum dots can be used to construct in vitro assays facilitating FRET. Not only for diagnosis but also for therapy, the quantum dots could be successfully utilized while carrying drug cargo into the target cell compartment, and the real time delivery of the drug molecule and its effectiveness could be monitored. In this way, the quantum dot FRET-based drug screening approaches could possibly be established. However, there is one important challenge to be addressed about their toxicity. In order to bear with the toxicity of the quantum dots, various attempts were made using cell-based experiments focused on the fate of the quantum dots within the cell, especially about distribution and accumulation of the quantum dots [101-103]. To overcome these disadvantages, strategies were tested to reduce toxicity of the quantum dots including negatively charged surface and biocompatible surface coatings. Another reported approach was based on using less toxic materials instead of $\mathrm{Cd}$ for the synthesis of semiconductor nanoparticles such as $\mathrm{ZnO}$ and $\mathrm{ZnSe}[104,105]$. 
To sum up, semiconductor colloidal quantum dots make one of the most popular and promising tools in biomedical and biochemical applications, potentially enabling game-changing detection and imaging systems for life sciences. Quantum dot-based FRET applications allow for the flexibility of carrying out detection and imaging studies at a wide range of wavelength choice at high efficiency levels with their broadband absorption and long fluorescence lifetimes, compared to the available organic dyes. With the ease of their surface functionalization, the colloidal quantum dots are capable of serving as efficient FRET donors for a wide range of applications with a potential high impact of emerging commercial production lines toward biomedical diagnostic and therapy tools.

\section{References}

1. Luo SL, Zhang EL, Su YP, Cheng TM et al (2011) A review of NIR dyes in cancer targeting and imaging. Biomaterials 32:7127-7138

2. Yuca E, Karatas AY, Seker UOS, Gungormus M et al (2011) In vitro labeling of hydroxyapatite minerals by an engineered protein. Biotechnol Bioeng 108:1021-1030

3. Sameiro M, Goncalves T (2009) Fluorescent labeling of biomolecules with organic probes. Chem Rev 109:190-212

4. Vendrell M, Lee JS, Chang YT (2010) Diversity-oriented fluorescence library approaches for probe discovery and development. Curr Opin Chem Biol 14:383-389

5. Margulies D, Hamilton AD (2010) Combinatorial protein recognition as an alternative approach to antibody-mimetics. Curr Opin Chem Biol 14:705-712

6. Sapsford KE, BertiI L, Medintz L (2006) Materials for fluorescence resonance energy transfer analysis: beyond traditional donor-acceptor combinations. Angew Chem Int Ed 45:4562-4588

7. Mutlugun E, Nizamoglu S, Demir HV (2009) Highly efficient nonradiative energy transfer using charged $\mathrm{CdSe} / \mathrm{ZnS}$ nanocrystals for light-harvesting in solution. Appl Phys Lett 95:033106

8. Krukenberg KA, Street TO, Lavery LA, Agard DA (2011) Conformational dynamics of the molecular chaperone Hsp90. Q Rev Biophys 44:229-255

9. Ratzke C, Mickler M, Hellenkamp B, Buchner J et al (2010) Dynamics of heat shock protein 90 C-terminal dimerization is an important part of its conformational cycle. Proc Natl Acad Sci USA 107:16101-16106

10. Huttunen R, Shweta ME, Lahdenranta M et al (2011) Single-label time-resolved luminescence assay for estrogen receptor-ligand binding. Anal Biochem 415:27-31

11. Rajdev P, Mondol T, Makhal A, Pal SK (2011) Simultaneous binding of anti-tuberculosis and anti-thrombosis drugs to a human transporter protein: a FRET study. J Photochem Photobiol B-Biol 103:153-158

12. Liu S, He J, Jin HL, Yang F et al (2011) Enhanced dynamic range in a genetically encoded $\mathrm{Ca}^{(2+)}$ sensor. Biochem Biophys Res Commun 412:155-159

13. Breton B, Sauvageau E, Zhou J, Bonin H et al (2010) Multiplexing of multicolor bioluminescence resonance energy transfer. Biophys J 99:4037-4046

14. Li M, Cushing SK, Wang QY, Shi XD et al (2011) Size-dependent energy transfer between $\mathrm{CdSe} / \mathrm{ZnS}$ quantum dots and gold nanoparticles. J Phys Chem Lett 2:2125-2129

15. Michalet X, Pinaud FF, Bentolila LA, Tsay JM et al (2005) Quantum dots for live cells, in vivo imaging, and diagnostics. Science 307:538-544

16. Burda C, Chen XB, Narayanan R, El-Sayed MA (2005) Chemistry and properties of nanocrystals of different shapes. Chem Rev 105:1025-1102

17. Rosi NL, Mirkin CA (2005) Nanostructures in biodiagnostics. Chem Rev 105:1547-1562 
18. Hildebrandt N (2011) Biofunctional quantum dots: controlled conjugation for multiplexed biosensors. ACS Nano 5:5286-5290

19. Mattoussi H (2010) In: Klimov VI (ed) Nanocrystal quantum dots, Ch 10. CRC Press, pp 369-393

20. Murray CB, Kagan CR, Bawendi MG (2000) Synthesis and characterization of monodisperse nanocrystals and close-packed nanocrystal assemblies. Annu Rev Mater Sci 30:545-610

21. Murray CB, Norris DJ, Bawendi MG (1993) Synthesis and characterization of nearly monodisperse Cde $(\mathrm{E}=\mathrm{S}, \mathrm{Se}, \mathrm{Te})$ semiconductor nanocrystallites. J Am Chem Soc 115:8706-8715

22. Pandey G, Dixit S (2011) Growth mechanism and optical properties determination of CdS nanostructures. J Phys Chem C 115:17633-17642

23. Miao SD, Hickey SG, Rellinghaus B, Waurisch C et al (2010) Synthesis and characterization of cadmium phosphide quantum dots emitting in the visible red to near-infrared. J Am Chem Soc 132:5613-5615

24. Rogach AL, Gaponik N (2008) In: Semiconductor nanocrystal quantum dots. Synthesis, assembly, spectroscopy and applications (Rogach AL ed). Springer, pp 73-99

25. Gupta S, Uhlmann P, Agrawal M, Lesnyak V et al (2008) Covalent immobilization of quantum dots on macroscopic surfaces using poly(acrylic acid) brushes. J Mater Chem $18: 214-220$

26. Gaponenko SV, Ozel T, Nizamoglu S, Sefunc MA et al (2011) Anisotropic emission from multilayered plasmon resonator nanocomposites of isotropic semiconductor quantum dots. ACS Nano 5:1328-1334

27. Sun HZ, Ning Y, Zhang H, Zhang JH et al (2009) Synthesis and characterization of CdTe nanoparticle/polymer functional composites. J Nanosci Nanotechno 9:7374-7378

28. Osovsky R, Shavel A, Gaponik N, Amirav L et al (2005) Electrostatic and covalent interactions in CdTe nanocrystalline assemblies. J Phys Chem B 109:20244-20250

29. Qiu T, Zhao D, Zhou G, Liang Y et al (2010) A positively charged QDs-based FRET probe for micrococcal nuclease detection. Analyst 135:2394-2399

30. Lee J, Choi Y, Kim J, Park E et al (2009) Positively charged compact quantum dot-DNA complexes for detection of nucleic acids. Chemphyschem 10:806-811

31. Kim YS, Jurng J (2011) Gold nanoparticle-based homogeneous fluorescent aptasensor for multiplex detection. Analyst 136:3720-3724

32. Lu H, Schops O, Woggon U, Niemeyer CM (2008) Self-assembled donor comprising quantum dots and fluorescent proteins for long-range fluorescence resonance energy transfer. J Am Chem Soc 130:4815-4827

33. He ZK, Qiu T, Zhao D, Zhou GH et al (2010) A positively charged QDs-based FRET probe for micrococcal nuclease detection. Analyst 135:2394-2399

34. Liu W, Howarth M, Greytak AB, Zheng Y et al (2008) Compact biocompatible quantum dots functionalized for cellular imaging. J Am Chem Soc 130:1274-1284

35. Mattoussi H, Susumu K, Uyeda HT, Medintz IL et al (2007) Enhancing the stability and biological functionalities of quantum dots via compact multifunctional ligands. J Am Chem Soc 129:13987-13996

36. Pinaud F, King D, Moore HP, Weiss S (2004) Bioactivation and cell targeting of semiconductor $\mathrm{CdSe} / \mathrm{ZnS}$ nanocrystals with phytochelatin-related peptides. J Am Chem Soc 126:6115-6123

37. Lee CM, Jang D, Cheong SJ, Kim EM et al (2010) Surface engineering of quantum dots for in vivo imaging. Nanotechnology 21:285102

38. Peelle BR, Krauland EM, Wittrup KD, Belcher AM (2005) Design criteria for engineering inorganic material-specific peptides. Langmuir 21:6929-6933

39. Grailhe R, Park HY, Kim K, Hong S et al (2010) Compact and versatile nickelnitrilotriacetate-modified quantum dots for protein imaging and forster resonance energy transfer based assay. Langmuir 26:7327-7333 
40. Medintz IL, Boeneman K, Mei BC, Dennis AM et al (2009) Sensing caspase 3 activity with quantum dot-fluorescent protein assemblies. J Am Chem Soc 131:3828-3829

41. Medintz IL, Boeneman K, Deschamps JR, Buckhout-White S et al (2010) Quantum dot DNA bioconjugates: attachment chemistry strongly influences the resulting composite architecture. ACS Nano 4:7253-7266

42. Kizek R, Ryvolova M, Chomoucka J, Janu L et al (2011) Biotin-modified glutathione as a functionalized coating for bioconjugation of CdTe-based quantum dots. Electrophoresis 32:1619-1622

43. Nikiforov TT, Beechem JM (2006) Development of homogeneous binding assays based on fluorescence resonance energy transfer between quantum dots and Alexa Fluor fluorophores. Anal Biochem 357:68-76

44. Terpe K (2003) Overview of tag protein fusions: from molecular and biochemical fundamentals to commercial systems. Appl Microbiol Biotechnol 60:523-533

45. Demir HV, Seker US, Ozel UOST (2011) Peptide-mediated constructs of quantum dot nanocomposites for enzymatic control of nonradiative energy transfer. Nano Lett 11:1530-1539

46. Willner I, Gill R, Zayats M (2008) Semiconductor quantum dots for bioanalysis. Angew Chem Int Ed 47:7602-7625

47. Cicek N, Nizamoglu S, Ozel T, Mutlugun E et al. (2009) Structural tuning of color chromaticity through nonradiative energy transfer by interspacing CdTe nanocrystal monolayers. Appl Phys Lett 94:061105

48. Krull UJ, Algar WR, Tavares AJ (2010) Beyond labels: a review of the application of quantum dots as integrated components of assays, bioprobes, and biosensors utilizing optical transduction. Anal Chim Acta 673:1-25

49. Su XG, Li YB, Ma Q, Wang XY (2007) Fluorescence resonance energy transfer between two quantum dots with immunocomplexes of antigen and antibody as a bridge. Luminescence 22:60-66

50. Su XG, Ma Q, Wang XY, Wan Y et al (2005) Fluorescence resonance energy transfer in doubly-quantum dot labeled IgG system. Talanta 67:1029-1034

51. MattoussiI H, Medintz L (2009) Quantum dot-based resonance energy transfer and its growing application in biology. Phys Chem Chem Phys 11:17-45

52. Lee EK, Wei OD, Lee M, Yu X et al (2006) Development of an open sandwich fluoroimmunoassay based on fluorescence resonance energy transfer. Anal Biochem 358:31-37

53. Zhao YD, Li YQ, Wang JH, Zhang HL et al (2010) High-sensitivity quantum dot-based fluorescence resonance energy transfer bioanalysis by capillary electrophoresis. Biosens Bioelectron 25:1283-1289

54. Goldman ER, Medintz IL, Whitley JL, Hayhurst A et al (2005) A hybrid quantum dotantibody fragment fluorescence resonance energy transfer-based TNT sensor. J Am Chem Soc 127:6744-6751

55. Rogach AL, Sukhanova A, Susha AS, Bek A et al (2007) Nanocrystal-encoded fluorescent microbeads for proteomics: antibody profiling and diagnostics of autoimmune diseases. Nano Lett 7:2322-2327

56. Zhou DJ, Piper JD, Abell C, Klenerman D et al. (2005) Fluorescence resonance energy transfer between a quantum dot donor and a dye acceptor attached to DNA. Chem Commun 38:4807-4809

57. Cady NC, Strickland AD, Batt CA (2007) Optimized linkage and quenching strategies for quantum dot molecular beacons. Mol Cell Probe 21:116-124

58. Wu CS, Cupps JM, Fan XD (2009) Compact quantum dot probes for rapid and sensitive DNA detection using highly efficient fluorescence resonant energy transfer. Nanotechnology 20:305502

59. Ozkan M, Kim JH, Chaudhary S (2007) Multicolour hybrid nanoprobes of molecular beacon conjugated quantum dots: FRET and gel electrophoresis assisted target DNA detection. Nanotechnology 18:195105 
60. Zhang CY, Yeh HC, Kuroki MT, Wang TH (2005) Single-quantum-dot-based DNA nanosensor. Nat Mater 4:826-831

61. Deng L, Chen Z, Li G, Zhang L et al (2008) A new method for the detection of ATP using a quantum-dot-tagged aptamer. Anal Bioanal Chem 392:1185-1188

62. Johnson LW, Zhang CY (2009) Single quantum-dot-based aptameric nanosensor for cocaine. Anal Chem 81:3051-3055

63. Oh MK, Kim GI, Kim KW, Sung YM (2009) The detection of platelet derived growth factor using decoupling of quencher-oligonucleotide from aptamer/quantum dot bioconjugates. Nanotechnology 20:175503

64. Zhou D, Ying L, Hong X, Hall EA et al (2008) A compact functional quantum dot-DNA conjugate: preparation, hybridization, and specific label-free DNA detection. Langmuir 24:1659-1664

65. Jiang G, Susha AS, Lutich AA, Stefani FD et al (2009) Cascaded FRET in conjugated polymer/quantum dot/dye-labeled DNA complexes for DNA hybridization detection. ACS Nano 3:4127-4131

66. Cheng AKH, Su HP, Wang A, Yu HZ (2009) Aptamer-based detection of epithelial tumor marker Mucin 1 with quantum dot-based fluorescence readout. Anal Chem 81:6130-6139

67. Wang LB, Xu LG, Zhu YY, Ma W et al (2011) Sensitive and specific DNA detection based on nicking endonuclease-assisted fluorescence resonance energy transfer amplification. J Phys Chem C 115:16315-16321

68. Carraway HE, Bailey VJ, Easwaran H, Zhang Y et al (2009) MS-qFRET: a quantum dotbased method for analysis of DNA methylation. Genome Res 19:1455-1461

69. Suzuki M, Husimi Y, Komatsu H, Suzuki K et al (2008) Quantum dot FRET Biosensors that respond to $\mathrm{pH}$, to proteolytic or nucleolytic cleavage, to DNA synthesis, or to a multiplexing combination. J Am Chem Soc 130:5720-5725

70. Bakalova R, Zhelev Z, Ohba H, Baba Y (2005) Quantum dot-conjugated hybridization probes for preliminary screening of siRNA sequences. J Am Chem Soc 127:11328-11335

71. Johnson LW, Zhang CY (2007) Quantifying RNA - peptide interaction by single-quantum dot-based nanosensor: an approach fair drug screening. Anal Chem 79:7775-7781

72. Johnson LW, Zhang CY (2006) Quantum dot-based fluorescence resonance energy transfer with improved FRET efficiency in capillary flows. Anal Chem 78:5532-5537

73. Sriram R, Yadav AR, Mace CR, Miller BL (2011) Validation of arrayed imaging reflectometry biosensor response for protein-antibody interactions: cross-correlation of theory, experiment, and complementary techniques. Anal Chem 83:3750-3757

74. Amniai L, Lippens G, Landrieu I (2011) Characterization of the AT180 epitope of phosphorylated Tau protein by a combined nuclear magnetic resonance and fluorescence spectroscopy approach. Biochem Biophys Res Commun 412:743-746

75. Garai K, Frieden CC (2010) The association-dissociation behavior of the ApoE proteins: kinetic and equilibrium studies. Biochemistry 49:9533-9541

76. Sarkar R, Narayanan SS, Palsson LO, Dias F et al (2007) Direct conjugation of semiconductor nanocrystals to a globular protein to study protein-folding intermediates. J Phys Chem B 111:12294-12298

77. Medintz IL, Deschamps JR (2006) Maltose-binding protein: a versatile platform for prototyping biosensing. Curr Opin Biotechnol 17:17-27

78. Medintz IL, Konnert JH, Clapp AR, Stanish I et al (2004) A fluorescence resonance energy transfer-derived structure of a quantum dot-protein bioconjugate nanoassembly. Proc Natl Acad Sci USA 101:9612-9617

79. Medintz IL, Clapp AR, Mattoussi H, Goldman ER et al (2003) Self-assembled nanoscale biosensors based on quantum dot FRET donors. Nat Mater 2:630-638

80. Chang E, Miller JS, Sun JT, Yu WW et al (2005) Protease-activated quantum dot probes. Biochem Biophys Res Commun 334:1317-1321 
81. Liu HY, Liang GX, Abdel-Halim ES, Zhu JJ (2011) A sensitive and selective quantum dots-based FRET biosensor for the detection of cancer marker type IV collagenase. Anal Methods 3:1797-1801

82. Pons T, Medintz IL, Sapsford KE, Higashiya S et al (2007) On the quenching of semiconductor quantum dot photoluminescence by proximal gold nanoparticles. Nano Lett 7:3157-3164

83. Shi LF, De Paoli V, Rosenzweig N, Rosenzweig Z (2006) Synthesis and application of quantum dots FRET-based protease sensors. J Am Chem Soc 128:10378-10379

84. Shi LF, Rosenzweig N, Rosenzweig Z (2007) Luminescent quantum dots fluorescence resonance energy transfer-based probes for enzymatic activity and enzyme inhibitors. Anal Chem 79:208-214

85. Sapsford KE, Granek J, Deschamps JR, Boeneman K et al (2011) Monitoring botulinum neurotoxin A activity with peptide-functionalized quantum dot resonance energy transfer sensors. ACS Nano 5:2687-2699

86. Prasuhn DE, Feltz A, Blanco-Canosa JB, Susumu K et al (2010) Quantum dot peptide biosensors for monitoring caspase 3 proteolysis and calcium ions. ACS Nano 4:5487-5497

87. Biswas P, Cella LN, Kang SH, Mulchandani A et al (2011) A quantum-dot based protein module for in vivo monitoring of protease activity through fluorescence resonance energy transfer. Chem Commun 47:5259-5261

88. Ghadiali JE, Cohen BE, Stevens MM (2010) Protein kinase-actuated resonance energy transfer in quantum dot-peptide conjugates. ACS Nano 4:4915-4919

89. Ghadiali JE, Lowe SB, Stevens MM (2011) Quantum-dot-based FRET detection of histone acetyltransferase activity. Angew Chem Int Ed 50:3417-3420

90. Xu CJ, Xing BG, Rao HH (2006) A self-assembled quantum dot probe for detecting betalactamase activity. Biochem Biophys Res Commun 344:931-935

91. Skajaa T, Zhao YM, van den Heuvel DJ, Gerritsen HC et al (2010) Quantum dot and Cy5.5 labeled nanoparticles to investigate lipoprotein biointeractions via forster resonance energy transfer. Nano Lett 10:5131-5138

92. Rakovich A, Sukhanova A, Bouchonville N, Lukashev E et al (2010) Resonance energy transfer improves the biological function of bacteriorhodopsin within a hybrid material built from purple membranes and semiconductor quantum dots. Nano Lett 10:2640-2648

93. Seker UOS, Ozel T, Demir HV (2011) Peptide-mediated constructs of quantum dot nanocomposites for enzymatic control of nonradiative energy transfer. Nano Lett 11:1530-1539

94. Harashima H, Shaheen SM, Akita H, Yamashita A et al (2011) Quantitative analysis of condensation/decondensation status of pDNA in the nuclear sub-domains by QD-FRET. Nucleic Acids Res 39:E48-U108

95. Bagalkot V, Zhang L, Levy-Nissenbaum E, Jon S et al (2007) Quantum dot - Aptamer conjugates for synchronous cancer imaging, therapy, and sensing of drug delivery based on Bi-fluorescence resonance energy transfer. Nano Lett 7:3065-3070

96. Kang WJ, Ko MH, Lee DS, Kim S (2009) Bioimaging of geographically adjacent proteins in a single cell by quantum dot-based fluorescent resonance energy transfer. Proteomics Clin Appl 3:1383-1388

97. Wang QL, Chen B, Liu P, Zheng MZ et al (2009) Calmodulin binds to extracellular sites on the plasma membrane of plant cells and elicits a rise in intracellular calcium concentration. J Biol Chem 284:12000-12007

98. Choi Y, Kim K, Hong S, Kim H et al (2011) Intracellular protein target detection by quantum dots optimized for live cell imaging. Bioconjug Chem 22:1576-1586

99. Srinivasan C, Siddiqui S, Silbart LK, Papadimitrakopoulos F et al (2009) Dual fluorescent labeling method to visualize plasmid DNA degradation. Bioconjug Chem 20:163-169

100. Delehanty JB, MattoussiI H, Medintz L (2009) Delivering quantum dots into cells: strategies, progress and remaining issues. Anal Bioanal Chem 393:1091-1105 
101. Su YY, Peng F, Jiang ZY, Zhong YL et al (2011) In vivo distribution, pharmacokinetics, and toxicity of aqueous synthesized cadmium-containing quantum dots. Biomaterials 32:5855-5862

102. Clift MJD, Brandenberger C, Rothen-Rutishauser B, Brown DM et al (2011) The uptake and intracellular fate of a series of different surface coated quantum dots in vitro. Toxicology 286:58-68

103. Qu Y, Li W, Zhou YL, Liu XF et al (2011) Full assessment of fate and physiological behavior of quantum dots utilizing Caenorhabditis elegans as a model organism. Nano Lett 11:3174-3183

104. Reddy KM, Feris K, Bell J, Wingett DG, et al. (2007) Selective toxicity of zinc oxide nanoparticles to prokaryotic and eukaryotic systems. Appl Phys Lett, 90:213903

105. Xie RG, Chen K, Chen XY, Peng XG (2008) InAs/InP/ZnSe Core/Shell/Shell quantum dots as near-infrared emitters: bright, narrow-band, non-cadmium containing, and biocompatible. Nano Res 1:457-464 